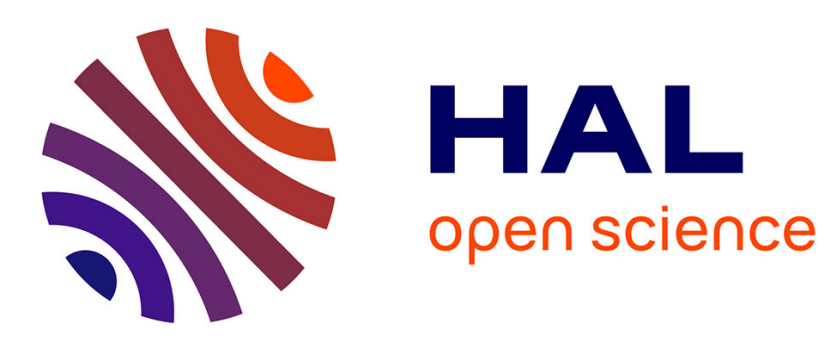

\title{
On the packing chromatic number of hypercubes
}

Pablo Torres, Mario Valencia-Pabon

\section{To cite this version:}

Pablo Torres, Mario Valencia-Pabon. On the packing chromatic number of hypercubes. Electronic Notes in Discrete Mathematics, 2013, 44 (5), pp.263-268. hal-00926875

\section{HAL Id: hal-00926875 https://hal.science/hal-00926875}

Submitted on 10 Jan 2014

HAL is a multi-disciplinary open access archive for the deposit and dissemination of scientific research documents, whether they are published or not. The documents may come from teaching and research institutions in France or abroad, or from public or private research centers.
L'archive ouverte pluridisciplinaire $\mathbf{H A L}$, est destinée au dépôt et à la diffusion de documents scientifiques de niveau recherche, publiés ou non, émanant des établissements d'enseignement et de recherche français ou étrangers, des laboratoires publics ou privés. 


\title{
On the packing chromatic number of hypercubes *
}

\author{
Pablo Torres ${ }^{\dagger}$ \\ Mario Valencia-Pabon ${ }^{\ddagger}$
}

\begin{abstract}
The packing chromatic number $\chi_{\rho}(G)$ of a graph $G$ is the smallest integer $k$ needed to proper color the vertices of $G$ in such a way that the distance in $G$ between any two vertices having color $i$ be at least $i+1$. Goddard et al. [8] found an upper bound for the packing chromatic number of hypercubes $Q_{n}$. Moreover, they compute $\chi_{\rho}\left(Q_{n}\right)$ for $n \leq 5$ leaving as an open problem the remaining cases. In this paper, we obtain a better upper bound for $\chi_{\rho}\left(Q_{n}\right)$ and we compute the exact value of $\chi_{\rho}\left(Q_{n}\right)$ for $6 \leq n \leq 8$.
\end{abstract}

Keywords: packing chromatic number, upper bound, hypercube graphs.

\section{Introduction}

We consider finite undirected graphs without loops or multiple edges. The concept of packing coloring comes from the area of frequency planning in wireless networks. This model emphasizes the fact that some frequencies might be used more sparely than the others. In graph terms, we ask for a partitioning of the vertex set of a graph $G$ into disjoint classes $V_{1}, \ldots, V_{k}$ (representing frequency usage) according to the following constraint. Each color class $V_{i}$ is an $i$-packing, i.e. a set of vertices with the property that any distinct pair $u, v \in V_{i}$ satisfies $\operatorname{dist}(u, v) \geq i+1$. Here, $\operatorname{dist}(u, v)$ is the distance between $u$ and $v$, i.e. the length of the shortest path in $G$ from $u$ to $v$.

Such partitioning into $k$ classes is called a packing $k$-coloring, even though it is allowed that some sets $V_{i}$ may be empty. The smallest integer $k$ for which exists a packing $k$-coloring of $G$ is called the packing chromatic number of $G$, and it is denoted by $\chi_{\rho}(G)$. The notion of packing chromatic number was established by Goddard et al. [8] under the name broadcast chromatic

\footnotetext{
*Supported by French CNRS PEPS Project "Combinatoire et invariants des graphes topologiques et de leurs généralisations", 2011-2013.

${ }^{\dagger}$ Universidad Nacional de Rosario \& CONICET, Argentina, (ptorres@fceia.unr.edu.ar)

${ }^{\ddagger}$ LIPN Université Paris-Nord \& INRIA Nancy - Grand Est, France, (valencia@lipn.univ-paris13.fr)
} 
number. The term packing chromatic number was introduced by Brešar et al. [4].

Much work has been devoted to the packing chromatic number of graphs ([2], [3], [4], [5], [6], [7], [8]). Fiala and Golovach [5] showed that determining the packing chromatic number is an NP-hard problem for trees. Goddard et al. [8] provided polynomial time algorithms for cographs and split graphs. Recently, Argiroffo et al. [2], [3] gave polynomial time algorithms for special subfamilies of trees, for partner limited graphs and for $(q, q-4)$ graphs.

In this paper, we are interested in bounding and, whenever possible, finding the packing chromatic number of hypercubes. For any $n \in \mathbb{Z}^{+}$, the $n$-dimensional hypercube, denoted $Q_{n}$, is the graph in which the vertices are all binary vectors of length $n$ (i.e., the set $\{0,1\}^{n}$ ), and two vertices are adjacent if and only if they differ in exactly one coordinate. Based on coding theory, Goddard et al. [8] gave an asymptotic result for the packing chromatic number of hypercubes. They proved that $\chi_{\rho}\left(Q_{n}\right) \sim\left(\frac{1}{2}-O\left(\frac{1}{n}\right)\right) 2^{n}$. More precisely, Goddard et al. [8] obtained that $\chi_{\rho}\left(Q_{n}\right) \leq 2+\left(\frac{1}{2}-\frac{1}{4 n}\right) 2^{n}$. In the same paper, they also computed $\chi_{\rho}\left(Q_{n}\right)$ for $1 \leq n \leq 5$, leaving as an open problem the remaining cases.

The diameter, $\operatorname{diam}(G)$, of a connected graph $G$ is the maximum distance between two vertices of $G$. The Cartesian product $G \square H$ of graphs $G$ and $H$ is the graph with vertex set $V(G) \times V(H)$, vertices $(g, h)$ and $\left(g^{\prime}, h^{\prime}\right)$ being adjacent whenever $g g^{\prime} \in E(G)$ and $h=h^{\prime}$, or $h h^{\prime} \in E(H)$ and $g=g^{\prime}$. Brešar et al. [4] obtained that if $G$ and $H$ are connected graphs on at least two vertices, $\chi_{\rho}(G \square H) \geq\left(\chi_{\rho}(G)+1\right)|H|-\operatorname{diam}(G \square H)(|H|-1)-1$. Moreover, if $H=K_{n}$, the complete graph on $n \geq 2$ vertices, then $\chi_{\rho}\left(G \square K_{n}\right) \geq$ $n \chi_{\rho}(G)-(n-1) \operatorname{diam}(G)$. It is well known that the binary hypercube $Q_{n}$ is isomorphic to the graph $Q_{n-1} \square K_{2}$. Then, we obtain directly the following lower bound for the packing chromatic number of $Q_{n}$.

Corollary 1. Let $n \geq 2$. Then, $\chi_{\rho}\left(Q_{n}\right) \geq 2 \chi_{\rho}\left(Q_{n-1}\right)-(n-1)$.

In this paper, we improve the upper bound found by Goddard et al. [8]. For this, we use elementary algebraic techniques in order to construct a packing coloring of $Q_{n}$, for $n \geq 4$. Furthermore, we obtain the exact values of $\chi_{\rho}\left(Q_{n}\right)$ for $n=6,7$ and 8 . Most of our results are given without proof for lack of space.

\section{Packing colorings of $Q_{n}$ : the upper bound}

The principal result of this section is the following :

Theorem 1. $\chi_{\rho}\left(Q_{n}\right) \leq 3+2^{n}\left(\frac{1}{2}-\frac{1}{2^{\left[\log _{2} n\right\rceil}}\right)-2\left\lfloor\frac{n-4}{2}\right\rfloor$, for $n \geq 4$.

In order to prove Theorem 1 , we derive a packing coloring of $Q_{n}$ with such a number of colors. For this, we use an algebraic approach for the purpose of construct $2-$ and 3 -packings for $Q_{n}$ as follows. 
Let $\Gamma$ be a group and $C$ a subset of $\Gamma$ closed under inverses and identity free. The Cayley graph $\operatorname{Cay}(\Gamma, C)$ is the graph with $\Gamma$ as its vertex set, two vertices $u$ and $v$ being joined by an edge if and only if $u^{-1} v \in C$. The set $C$ is then called the connector set of $\operatorname{Cay}(\Gamma, C)$. It is well known that $Q_{n}$ is the Cayley graph of the Abelian group $\mathbb{Z}_{2}^{n}$ (the elements of $\mathbb{Z}_{2}^{n}$ are the binary $n$-vectors of the set $\{0,1\}^{n}$ and the group operation is the sum modulo two coordinatewise), where the connector set is the subset of $n$-vectors having exactly one coordinate equal to 1 . So, in order to construct some packings for $Q_{n}$ we will analyze the structure of a special subgroup of $\mathbb{Z}_{2}^{n}$.

Let $v \in \mathbb{Z}_{2}^{n}$. We denote by $v^{i}$ the $i^{\text {th }}$ coordinate of $v$, for $1 \leq i \leq n$, that is, $v=\left(v^{1}, \ldots, v^{i}, \ldots, v^{n}\right)$, where $v^{i}$ is either 0 or 1 .

Definition 1. Let $n \geq 4$. For $m \in\{4, \ldots, n\} \backslash\left\{2^{k}+1: k \in \mathbb{Z}^{+}\right\}$, consider the $n$-vectors of $\mathbb{Z}_{2}^{n}$ defined as follows :

1. Type I: If $m$ is even,

$$
v_{m}^{i}= \begin{cases}1, & \text { if } i \in\{1,2, m-1, m\} \\ 0, & \text { o.w. }\end{cases}
$$

2. Type II: If $m$ is odd and $m-1$ is not a power of 2 ,

$$
v_{m}^{i}= \begin{cases}1, & \text { if } i \in\left\{1, m-2^{\left\lfloor\log _{2} m\right\rfloor}, 1+2^{\left\lfloor\log _{2} m\right\rfloor}, m\right\} \\ 0, & \text { o.w. }\end{cases}
$$

We define the subset $W_{n} \subset \mathbb{Z}_{2}^{n}$ as the set containing the vectors $v_{m}$ defined previously.

Notice that every vector in $W_{n}$ has exactly four coordinates equal to 1. Moreover, by construction, we have that :

Remark 1. Let $n \geq 4$. Then, $\left|W_{n}\right|+1=\left|W_{n+1}\right|$ if and only if $n$ is not a power of two, otherwise $\left|W_{n}\right|=\left|W_{n+1}\right|$. Moreover, if $n$ is even (resp. odd), the new vector in $W_{n+1}$ is of Type II (resp. I).

Remark 2. Let $n \geq 4$. Notice that the distance between any pair of $n$ vectors in $W_{n}$ is even.

Besides, by induction on $n$, we can prove the following lemma.

Lemma 1. Let $n \geq 4$. Then, $\left|W_{n}\right|=n-1-\left\lceil\log _{2} n\right\rceil$.

Now, by computing the join of all the 2-subgroups generated by each one of the elements in $W_{n}$, we can deduce the following result.

Lemma 2. Let $n \geq 4$, and let $\mathcal{G}_{n}$ be the subgroup of the Abelian group $\mathbb{Z}_{2}^{n}$ generated by $W_{n}$. Then, the order of $\mathcal{G}_{n}$ is equal to $2^{\left|W_{n}\right|}$. 
The subgroup $\mathcal{G}_{n}$ plays an important role in the search of $i$-packings. In particular, we prove that any pair of vertices in $\mathcal{G}_{n}$ are at distance greater than 3 in $Q_{n}$, i.e. the set $\mathcal{G}_{n}$ is a 3-packing of $Q_{n}$. To obtain this, we use the structure in the construction of the sets $W_{n}$ and we proceed by induction on $n$.

Lemma 3. Let $n \geq 4$. For any $u, v \in Q_{n}, u \neq v$, such that $u, v \in \mathcal{G}_{n}$, we have that the distance between $u$ and $v$ in $Q_{n}$ verifies : $\operatorname{dist}(u, v) \geq 4$. Moreover, each element of $\mathcal{G}_{n}$ has an even number of coordinates (0 included) equal to 1.

Definition 2. Let $n \geq 4$. For each $j$, with $1 \leq j \leq\left\lfloor\frac{n-4}{2}\right\rfloor$, let $A_{j}$ and $B_{j}$ be 2 -subsets of $\mathbb{Z}_{2}^{n}$ constructed as follows : $A_{j}$ is formed by two n-vectors $a_{j_{1}}$ and $a_{j_{2}}$ where $a_{j_{1}}$ has only one 1 in coordinate $j+2$ and 0 otherwise, and $a_{j_{2}}$ has 1 in coordinates $t \in[2 j+4] \backslash\{j+2\}$, and 0 otherwise. The 2 -set $B_{j}$ is formed by two n-vectors $b_{j_{1}}$ and $b_{j_{2}}$, where $b_{j_{1}}$ (resp. $b_{j_{2}}$ ) is equal to $a_{j_{1}}$ (resp. $a_{j_{2}}$ ) but with the two first coordinates complemented.

The following result can be easily deduced.

Lemma 4. Let $n \geq 6$. Let $g_{2} \in \mathbb{Z}_{2}^{n}$ (resp. $g_{3} \in \mathbb{Z}_{2}^{n}$ ) be the element having only the first coordinate (resp. the second coordinate) equal to 1 and the remaining coordinates equal to 0 . For each $j$, with $1 \leq j \leq\left\lfloor\frac{n-4}{2}\right\rfloor$, the 2 -sets $A_{j}$ and $B_{j}$ in Definition 2 verify the following properties:

1. $A_{j} \cap\left(g_{2}+\mathcal{G}_{n}\right)=A_{j} \cap\left(g_{3}+\mathcal{G}_{n}\right)=B_{j} \cap\left(g_{2}+\mathcal{G}_{n}\right)=B_{j} \cap\left(g_{3}+\mathcal{G}_{n}\right)=\emptyset$.

2. $A_{j}$ (resp. $\left.B_{j}\right)$ is a $(2 j+2)$-packing (resp. $(2 j+3)$-packing) of $Q_{n}$.

From the previous lemmas, we are able to prove Theorem 1.

\section{Proof of Theorem 1}

Let $n \geq 4$. Let $\mathcal{G}_{n}$ be the subgroup of the Abelian group $\mathbb{Z}_{2}^{n}$ generated by the set $W_{n}$ (see Definition 1). Clearly, the elements of $\mathbb{Z}_{2}^{n}$ correspond to the vertices of the binary $n$-dimensional hypercube $Q_{n}$. Let $I_{1}^{n}, I_{2}^{n}$ and $I_{3}^{n}$ be subsets of vertices of $Q_{n}$ constructed as follows : $I_{1}^{n}$ is the set of all vertices in $Q_{n}$ having an even number (0 included) of coordinates equal to 1 . The sets $I_{2}^{n}$ and $I_{3}^{n}$ are the cosets $g_{2}+\mathcal{G}_{n}$ and $g_{2}+\mathcal{G}_{n}$, resp., where $g_{2}$ and $g_{3}$ are defined as in Lemma 4. By Lemma 3, it is clear that both $I_{2}^{n}$ and $I_{3}^{n}$ are disjoint of $I_{1}^{n}$, because all the elements in $I_{2}^{n}$ and in $I_{3}^{n}$ have an odd number of coordinates equal to 1 . Moreover, $I_{2}^{n}$ and $I_{3}^{n}$ are disjoint by the Lagrange's Theorem. Therefore, $I_{i}^{n}$ is an $i$-packing of $Q_{n}$, for $i=1,2,3$. Now, observe that the family of sets in Definition 2 are pairwise disjoint. Furthermore, by Lemma 4 , for $1 \leq j \leq\left\lfloor\frac{n-4}{2}\right\rfloor$, the sets $A_{j}$ and $B_{j}$ are $(2 j+2)$-packing and $(2 j+3)$-packing of $Q_{n}$ respectively. By given a different color greater than $2\left\lfloor\frac{n-4}{2}\right\rfloor+3$ to the remaining vertices in $Q_{n}$, we obtain the desired packing coloring of $Q_{n}$ with $3+2^{n}\left(\frac{1}{2}-\frac{1}{2^{\left[\log _{2} n\right\rceil}}\right)-2\left\lfloor\frac{n-4}{2}\right\rfloor$ colors. 


\section{Lower bounds for $\chi_{\rho}\left(Q_{n}\right)$ : the cases $n=6,7$ and 8}

As mentioned in the introduction, Goddard et al. [8] computed the packing chromatic numbers of the first five hypercubes: $\chi_{\rho}\left(Q_{1}\right)=2, \chi_{\rho}\left(Q_{2}\right)=3$, $\chi_{\rho}\left(Q_{3}\right)=5, \chi_{\rho}\left(Q_{4}\right)=7$ and $\chi_{\rho}\left(Q_{5}\right)=15$. Moreover, by Corollary 1 and Theorem 1, we know that $25 \leq \chi_{\rho}\left(Q_{6}\right) \leq 25,44 \leq \chi_{\rho}\left(Q_{7}\right) \leq 49$ and $89 \leq \chi_{\rho}\left(Q_{8}\right) \leq 95$. Thus, we have the following direct result.

Corollary 2. $\chi_{\rho}\left(Q_{6}\right)=25$

The main result in this section is the computation of a tight lower bound for the packing chromatic number of hypercubes of dimension 7 and 8 . In order to obtain these lower bounds, we combine the results obtained by Agrell et al. [1] concerning the size of the maximum number of vertices that can be covered by subsets of $i$-packings in $Q_{7}$ (resp. $Q_{8}$ ), for $1 \leq i<7$ (resp. $1 \leq i<8$ ), with the ones concerning the maximum size of balanced independent (and dominating) sets on hypercubes obtained by Ramras [9]. Thus, we obtain that,

Theorem 2. $\chi_{\rho}\left(Q_{7}\right) \geq 49$ and $\chi_{\rho}\left(Q_{8}\right) \geq 95$.

Therefore, by Theorems 1 and 2, we conclude that $\chi_{\rho}\left(Q_{7}\right)=49$ and $\chi_{\rho}\left(Q_{8}\right)=95$.

\section{References}

[1] E. Agrell, A. Vardy, and K. Zeger. A table of upper bounds for binary codes. IEEE Trans. Inform. Theory, 47(7):3004-3006, 2001.

[2] G. Argiroffo, G. Nasini, P. Torres. The packing coloring problem for lobsters and partner limited graphs, Discrete Applied Mathematics (2012), doi:10.1016/j.dam.2012.08.008.

[3] G. Argiroffo, G. Nasini, P. Torres. The packing coloring problem for $(q, q-4)$ graphs, to appear in Lecture Notes in Computer Science, 2012 .

[4] B. Brešar, S. Klavžar, D.F. Rall. On the packing chromatic numbers of cartesian products, hexagonal lattice, and trees, Discrete Applied Mathematics, 155 (2007) 2303-2311.

[5] J. Fiala, P. A. Golovach. Complexity of the packing coloring problem of trees, Discrete Applied Mathematics, 158 (2010), 771-778.

[6] J. Fiala, S. Klavžar, B. Lidicky. The packing chromatic number of infinite product graphs, European J. of Combin., 30 (2009) 1101-1113. 
[7] A. Finbow, D. F. Rall. On the packing chromatic number of some lattices, Discrete Applied Mathematics, 158 (2010) 1224-1228.

[8] W. Goddard, S. M. Hedetniemi, S. T. Hedetniemi, J. Harris, D. F. Rall. Broadcast Chromatic Numbers of Graphs, Ars Combinatoria, 86 (2008) $33-49$.

[9] M. Ramras. Balanced independent sets in hypercubes, Australasian J. of Combinatorics, 48 (2010) 57-72. 